\title{
Metformin improves nonalcoholic fatty liver disease in obese mice via down-regulation of apolipoprotein A5 as part of the AMPK/LXRa signaling pathway
}

\author{
Min-Jie Lin', ${ }^{1}$, Wen Dai ${ }^{2, *}$, Melanie J. Scott ${ }^{3}$, Rong Li $^{4}$, Yi-Qi Zhang ${ }^{2}$, Yang Yang ${ }^{2}$, \\ Lu-Zhu Chen ${ }^{2}$ and Xian-Sheng Huang ${ }^{2}$ \\ ${ }^{1}$ Clinical Skills Training Center, The Second Xiangya Hospital, Central South University, Changsha, Hunan, 410011, China \\ ${ }^{2}$ Department of Cardiovascular Medicine, The Second Xiangya Hospital, Central South University, Changsha, Hunan, 410011, \\ China \\ ${ }^{3}$ Department of Surgery Labs, University of Pittsburgh, Pittsburgh, PA 15213, USA \\ ${ }^{4}$ Department of Stomatology, The Second Xiangya Hospital, Central South University, Changsha, Hunan, 410011, China \\ *These authors have contributed equally to this work \\ Correspondence to: Xian-Sheng Huang, email: initialhxs@163.com \\ Keywords: triglyceride; hepatocytes; lipid droplets; leptin-deficient; ob/ob mice \\ Received: August 11,2017 Accepted: September 22, $2017 \quad$ Published: October 30, 2017 \\ Copyright: Lin et al. This is an open-access article distributed under the terms of the Creative Commons Attribution License 3.0 \\ (CC BY 3.0), which permits unrestricted use, distribution, and reproduction in any medium, provided the original author and source \\ are credited.
}

\section{ABSTRACT}

Apolipoprotein A5 (apoA5) has been implicated in the formation of hepatocyte lipid droplets, a histological hallmark of non-alcoholic fatty liver disease (NAFLD). Recent evidence demonstrated that liver $X$ receptor a ( $L X R a)$, a transcription factor involved in down-regulation of APOA5 mRNA, is activated by AMP-activated protein kinase (AMPK) that contributes to metformin-related antihyperglycemic effects. In this study we investigated the role of apoA5 and AMPK/LXRa signaling pathway in metformin-related improvement of NAFLD. Leptin-deficient (ob/ob) obese mice with NAFLD were treated with metformin, and signaling pathways were compared with non-metformin treated mice. Additionally, we determined cellular apoA5 and triglyceride (TG) levels in mouse hepatocytes in vitro and the effects of metformin, with or without an AMPK inhibitor or LXRa siRNA, on these levels. We found that metformin dose-dependently ameliorated hepatosteatosis and liver dysfunction in $o b / o b$ mice, with a significant reduction in hepatic apoA5 expression and TG level. Metformin also dose-dependently increased phosphorylation of hepatic AMPK and LXRa in ob/ob mice. Similarly, metformin decreased apoA5 expression and TG level in mouse hepatocytes, with increased phosphorylation of cellular AMPK and LXRa. Addition of AMPK inhibitor or siRNA knockdown of LXRa significantly attenuated metformin-induced down-regulation of cellular apoA5 expression and TG level. AMPK inhibitor also significantly inhibited metformin-induced LXRa phosphorylation in these hepatocytes. Therefore, our findings indicate that metformin improves obesity-related NAFLD via inhibition of hepatic apoA5 synthesis as part of the AMPK/LXRa signaling pathway.

\section{INTRODUCTION}

Non-alcoholic fatty liver disease (NAFLD) represents the most common chronic liver disorder, and is strongly linked to obesity with a reported prevalence as high as $80 \%$ in obese population $[1,2]$. For obese patients, NAFLD is frequently accompanied by hypertriglyceridemia [3]. NAFLD and 
hypertriglyceridemia are actually two sides of the same coin, because they both result from disordered triglyceride (TG) metabolism in two interrelated compartments: liver and the plasma [3]. Recent evidence demonstrated that metformin, a first-line antihyperglycemic drug for type 2 diabetes mellitus, effectively improved liver dysfunction in obese patients with NAFLD [4], which indicates that this agent could be enlisted as a promising medicine for obesity-related NAFLD. However, the underlying mechanism of metformin's effects in NAFLD remain to be elucidated.

Interestingly, accumulated data implicate the potential role of apolipoprotein A5 (apoA5) in the crosstalk between hypertriglyceridemia and NAFLD. Earlier studies implicated apoA5 as a key regulator of plasma TG homeostasis [5, 6], while recent data identified its role in the biogenesis of hepatocyte lipid droplets [79], a histological hallmark of NAFLD. More recently, two studies observed overexpression of hepatic APOA5 mRNA in NAFLD patients, which implicated apoA5 as a potential therapeutic target for NAFLD $[10,11]$. APOA5 mRNA expression is regulated by several factors including liver $\mathrm{X}$ receptor $\alpha(\mathrm{LXR} \alpha)$, a ligand-activated nuclear transcription factor that has been demonstrated to downregulate hepatic APOA5 mRNA expression [12]. Notably, LXR $\alpha$ activation by phosphorylated AMP-activated protein kinase (AMPK), a key factor for pharmacological actions of metformin, has recently been demonstrated to contribute to the antihyperglycemic effects of metformin [13]. Considering the fact that apoA5 is a target gene of $\mathrm{LXR} \alpha$ [12], apoA5 could be a potential contributor to metformin-mediated protection against NAFLD. Here, we investigated whether metformin could improve obesity-related NAFLD through inhibition of hepatic apoA5 synthesis regulated via the AMPK/LXR $\alpha$ signaling pathway.

\section{RESULTS}

\section{Effects of metformin on energy intake, body weight, lipids, apoA5, glucose, insulin, HOMA- IR, and aminotransferases in $o b / o b$ mice}

As shown in Table 1, compared to controls, $o b /$ $o b$ mice in the NAFLD group had significant elevations in energy intake, body weight, lipids, glucose, insulin, the homeostasis model assessment of insulin resistance (HOMA-IR), alanine aminotransferase (ALT), and aspartate aminotransferase (AST). However, metformin effectively decreased these levels in a dose-dependent manner. Interestingly, higher apoA5 levels were observed in NAFLD group than controls, whereas metformin dosedependently decreased apoA5 levels of these $o b / o b$ mice, but these levels were not reduced to control levels.

To identify the association of apoA5 with body weight, lipids, HOMA-IR, and liver function (ALT), we performed correlation analyses. After pooling all data of each group, our correlation analyses showed that apoA5 levels were positively correlated with TG $(\mathrm{r}=0.58, p$ $<0.01)$, body weight $(\mathrm{r}=0.50, p<0.05)$, HOMA-IR ( $\mathrm{r}$ $=0.52, p<0.05)$, and ALT $(\mathrm{r}=0.53, p<0.05)$, but no significant correlation was found between apoA5 and cholesterol $(p>0.05)$. In addition, a significantly positive correlation was also observed between TG and weight $(\mathrm{r}=$ $0.60, p<0.01)$, body weight and HOMA-IR $(\mathrm{r}=0.62, p<$ $0.001)$, HOMA-IR and ALT $(r=0.56, p<0.05)$.

\section{Effects of metformin on hepatic steatosis, TG, apoA5, and phosphorylation of AMPK and LXRa in $o b / o b$ mice}

Typical hepatosteatosis, characterized by unstained areas consisting of fat vacuoles on $\mathrm{H} \& \mathrm{E}$, was observed in livers of NAFLD $o b / o b$ mice. Metformin dosedependently ameliorated hepatosteatosis of these $o b / o b$ mice as indicated by a reduction of fat vacuoles (Figure 1A). Steatosis scores in NAFLD mice were significantly higher than controls, and metformin dose-dependently decreased steatosis scores in $o b / o b$ mice (all $p<0.05$ ) (Figure 1B). Compared with controls, hepatic TG level in NAFLD mice were considerably elevated $(10.78 \pm 0.98$ vs. $45.56 \pm 4.76 \mu \mathrm{g} / \mathrm{mg}$ liver protein, $p<0.01)$. Metformin also effectively decreased hepatic TG level in a dosedependent manner $(26.63 \pm 2.85$ vs. $18.45 \pm 2.06 \mu \mathrm{g} / \mathrm{mg}$ liver protein, $p<0.05$ ) (Figure $1 \mathrm{C}$ ). Hepatic apoA5 protein expression in NAFLD mice were significantly elevated compared with control mice, and metformin similarly dose-dependently reduced hepatic apoA5 expressions of these $o b / o b$ mice (all $p<0.05$ ) (Figure 1D).

We also determined phosphorylation of hepatic AMPK and LXR $\alpha$ in these mice. This was assessed by determining the ratio of phosphorylated (p-AMPK/p$\mathrm{LXR} \alpha$ ) to total AMPK/LXR $\alpha$ expression. Although total AMPK expression was not changed, hepatic AMPK phosphorylation of $o b / o b$ mice in NAFLD group was lower than controls, and metformin dose-dependently increased AMPK phosphorylation (Figure 1E). Similarly, hepatic $\mathrm{LXR} \alpha$ phosphorylation in NAFLD mice was suppressed, while metformin dose-dependently enhanced LXR $\alpha$ phosphorylation of $o b / o b$ mice (Figure 1F).

\section{Effects of metformin on TG, apoA5, and phosphorylation of AMPK and LXR $\alpha$ in hepatocytes}

Compared to controls, cellular TG level in metformin-treated cells were significantly reduced $(40.59 \pm 4.25$ vs. $25.02 \pm 2.98 \mu \mathrm{g} / \mathrm{mg}$ cell protein, $p<$ 0.01). However, cellular TG level in AMPK inhibitortreated and LXR $\alpha$ knockdown (KD) cells were notably increased compared with metformin-treated cells. Cellular TG levels in LXR $\alpha$ KD hepatocytes was higher than in 
Table 1: Energy intake, body weight, lipids, apoA5, glucose, insulin, HOMA-IR, ALT and AST of mice after four weeks of high fat diet with or without low/high dose metformin

\begin{tabular}{lcccc}
\hline & Control & NAFLD & Low-dose metformin & High-dose metformin \\
\hline Energy intake (kcal) & $13.28 \pm 1.59$ & $19.60 \pm 2.35^{1}$ & $16.82^{2} \pm 2.02^{1,2}$ & $15.48 \pm 1.85^{1,2,3}$ \\
Body weight (g) & $25.12 \pm 1.56$ & $57.26 \pm 3.07^{1}$ & $45.76 \pm 2.60^{1,2}$ & $40.05 \pm 2.35^{1,2,3}$ \\
Cholesterol (mmol/L) & $2.68 \pm 0.22$ & $6.05 \pm 0.43^{1}$ & $4.86 \pm 0.35^{1,2}$ & $4.25 \pm 0.32^{1,2,3}$ \\
TG (mmol/L) & $1.20 \pm 0.09$ & $3.12 \pm 0.35^{1}$ & $2.35 \pm 0.26^{1,2}$ & $2.04 \pm 0.22^{1,2,3}$ \\
ApoA5 (ng/mL) & $112.50 \pm 13.48$ & $272.40 \pm 29.77^{1}$ & $218.05 \pm 24.25^{1,2}$ & $192.68^{1,2} \pm 20.89^{1,2,3}$ \\
Glucose (mmol/L) & $6.24 \pm 0.33$ & $17.14 \pm 0.87^{1}$ & $13.79 \pm 0.72^{1,2}$ & $10.97^{1,2} \pm 0.55^{1,2,3}$ \\
Insulin (mU/L) & $10.05 \pm 0.42$ & $23.45 \pm 1.18^{1}$ & $16.88^{1,2} \pm 0.85^{1,2}$ & $13.83 \pm 0.70^{1,2,3}$ \\
HOMA-IR & $2.79 \pm 0.13$ & $17.82 \pm 0.91^{1}$ & $10.35 \pm 0.52^{1,2}$ & $6.76 \pm 0.34^{1,2,3}$ \\
ALT (U/L) & $24.52 \pm 2.23$ & $162.59 \pm 8.12^{1}$ & $83.09 \pm 3.89^{1,2}$ & $71.38 \pm 3.62^{1,2,3}$ \\
AST (U/L) & $20.78 \pm 1.89$ & $180.62 \pm 9.83^{1}$ & $118.63 \pm 5.75^{1,2}$ & $79.29 \pm 4.02^{1,2,3}$ \\
\hline
\end{tabular}

1,2,3 Values were significantly different from control, NAFLD and low-dose metformin group, respectively $(p<0.05)$.

AMPK inhibitor-treated cells $(56.29 \pm 6.09$ vs. $49.95 \pm$ $5.35 \mu \mathrm{g} / \mathrm{mg}$ cell protein, $p<0.05$ ) (Figure $2 \mathrm{~A}$ ). Similarly, metformin resulted in a down-regulation of apoA5 expression in hepatocytes, but AMPK inhibitor or LXR $\alpha$ siRNA effectively attenuated metformin-induced reduction of apoA5 expression, and this effect was stronger in LXR $\alpha$ $\mathrm{KD}$ cells than in AMPK inhibitor-treated cells (all $p<$ 0.05) (Figure 2B).

We also investigated phosphorylation of hepatocyte AMPK and LXR $\alpha$ in vitro. As expected, metformin effectively increased AMPK phosphorylation in hepatocytes. When added with the AMPK inhibitor, the metformin-induced phosphorylation of hepatocyte AMPK was significantly repressed, while no inhibitory effect was observed in LXR $\alpha$ KD group (Figure 2C). Of note, LXR $\alpha$ phosphorylation in cells given metformin only was higher than control, which indicated metformin can activate LXR $\alpha$ in hepatocytes. By contrast, AMPK inhibitor and LXR $\alpha \mathrm{KD}$ attenuated metformin-induced up-regulation of cell $\mathrm{LXR} \alpha$, and this inhibitory effect was more notable in LXR $\alpha$ KD group (Figure 2D).

\section{DISCUSSION}

In this study we first investigated the effects of metformin on NAFLD in $o b / o b$ obese mice, which develop hyperglycemia, insulin resistance (IR), hypertriglyceridemia and fatty livers owing to an inherited deficiency of the appetite-suppressing hormone, leptin [14]. In this study, all $o b / o b$ mice developed typical hepatosteatosis, characterized by enriched hepatocyte lipid droplets, hepatic macro- and micro-steatosis, and hypertrophy, suggesting that our obesity-related NAFLD mouse models were successfully established. Interestingly, metformin not only effectively ameliorated hyperglycemia, insulin resistance and hypertriglyceridemia, but also markedly alleviated hepatosteatosis and liver dysfunction in $o b / o b$ mice. Therefore, our results demonstrate the beneficial effects of metformin on obesity-related NAFLD, in agreement with previously published data [4, 14]. Consistently with previous pre-clinical $[15,16]$ and clinical investigations $[17,18,19]$, the weight reducing effect of metformin was also revealed in our study. Such weight reduction itself or in combination with other mechanisms of metformin may synergistically contribute to improvement of NAFLD [20]. However, metformin has a modest effect on body weight [21] and the reducing effect of the drug is probably too small to provide a significant hepatic effect [20].

To date, the mechanism underlying the pharmacological actions of metformin on NAFLD remain incompletely understood, but it has been reported that metformin-mediated IR inhibition is partially responsible for its protection against NAFLD [4, 14]. Similarly, our study showed that metformin substantially decreased HOMA-IR (an index of insulin resistance) and plasma ALT levels (an index of liver dysfunction), both being positively correlated with each other, in these $o b / o b$ mice. Collectively, these data identified the contribution of metformin-mediated insulin resistance inhibition in amelioration of NAFLD.

A role for apoA5 in metformin-related amelioration of NAFLD was also identified by this study. As mentioned previously, apoA5 is an important player in intrahepatic TG homeostasis, where it promotes the formation of hepatocyte lipid droplets [7-9], a histological hallmark of NAFLD. Similarly, simultaneously elevated TG contents and apoA5 expression within the livers were detected in our $o b / o b$ mice with NAFLD. Together with our previous findings that APOA5 KD resulted in a significant decrease of hepatocyte TG level [22], our data demonstrate the crucial role of apoA5 in intrahepatic 


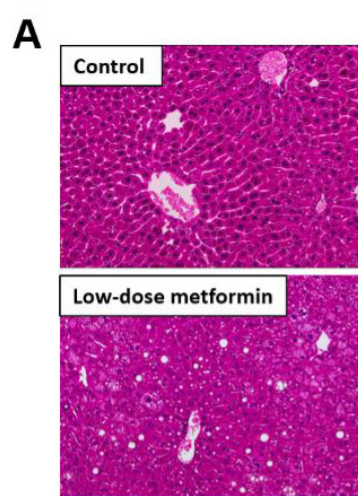

D
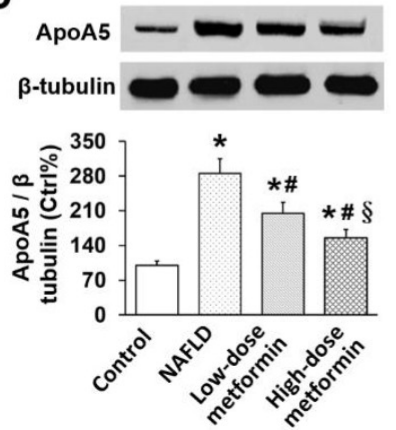

B

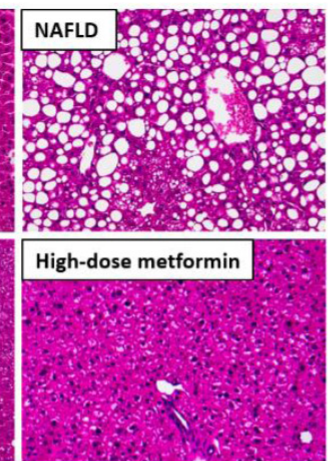

E
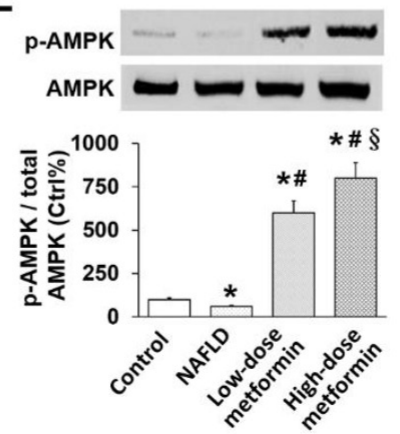

C

Hepatic TG contents

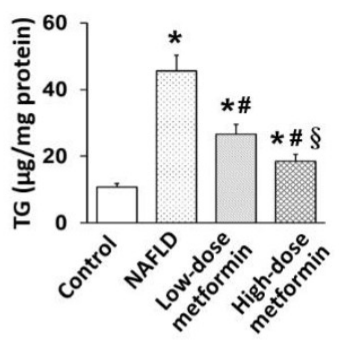

$\mathbf{F}$

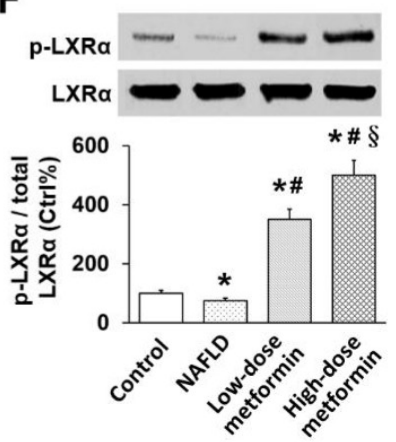

Figure 1: Effects of metformin on hepatic steatosis, TG, apoA5, and phosphorylation of AMPK and LXRa in mice. (A) H\&E histology of liver tissue ( $\times 400$ magnification); (B) Hepatic steatosis score; (C) Hepatic TG level; (D) Hepatic apoA5 protein levels; (E) Hepatic AMPK phosphorylation; (F) Hepatic LXR $\alpha$ phosphorylation. ${ }^{*} p<0.05$ vs. control; ${ }^{*} p<0.05$ vs. NAFLD group; ${ }^{\S} p<0.05$ vs. low-dose metformin group.

A

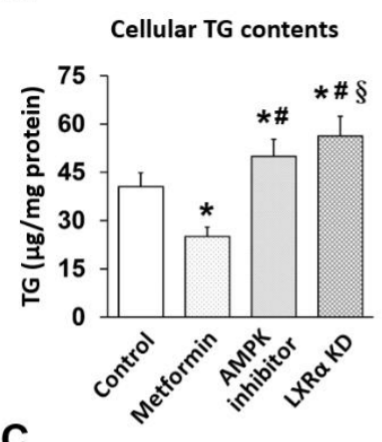

C

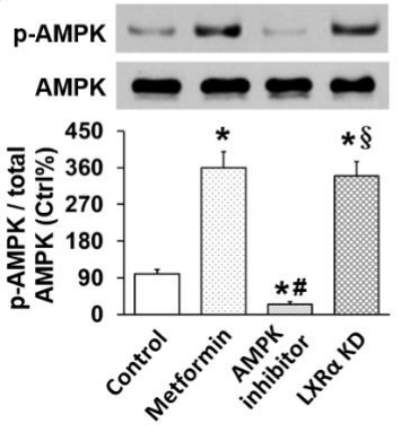

B

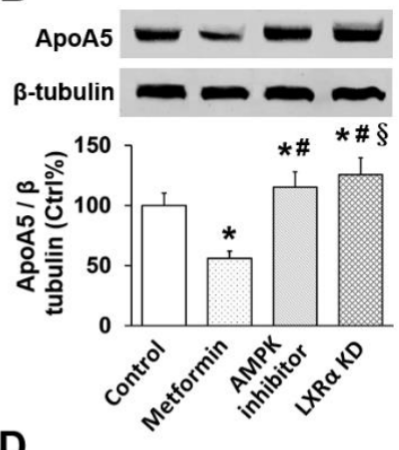

D

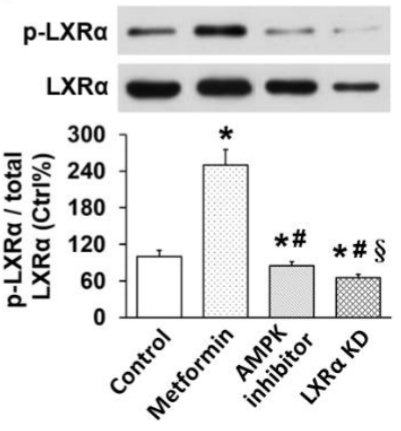

Figure 2: Effects of metformin on TG, apoA5, and phosphorylation of AMPK and LXR $\alpha$ in mouse hepatocytes. (A) Cellular TG level; (B) Cellular apoA5 protein levels; (C) Cellular AMPK phosphorylation; (D) Cellular LXR $\alpha$ phosphorylation. * $p<0.05$ vs. control; $; p<0.05$ vs. metformin group; ${ }^{\S} p<0.05$ vs. AMPK inhibitor group. 
TG accumulation. Pathologically, excessive intrahepatic TG accumulation inevitably leads to NAFLD [23], indicating the potential role of apoA5 in the pathogenesis of NAFLD, which has recently also been identified by two independent research groups [10, 11]. Ress et al observed an elevation of hepatic APOA5 mRNA in obese patients with NAFLD, which were markedly down-regulated after improvement in hepatosteatosis [10]. Similarly, Feng et al detected increased expression of APOA5 mRNA in pediatric NAFLD livers that was positively associated with increased hepatic TG level. They furthermore found an increase of hepatic APOA5 mRNA in obese rats with NAFLD [11]. Similarly, our study demonstrated that obese mice with NAFLD had higher hepatic apoA5 expression and TG level, and metformin treatment resulted in a considerable reduction of both hepatic apoA5 expression and TG level in livers of these animals. Moreover, metformin simultaneously decreased apoA5 expression and TG level in mouse hepatocytes in vitro. Together with our findings that metformin effectively ameliorated hepatosteatosis of these obese mice, we conclude that metformin protection against NAFLD is associated with its inhibition of hepatic apoA5 production.

We also made another interesting finding about plasma apoA5 and TG in this study. Plasma apoA5 levels were increased in $o b / o b$ mice despite their hypertriglyceridemic state, and metformin simultaneously decreased plasma apoA5 and TG levels. Additionally, a positive correlation between the two factors was identified in this study. These findings are consistent with data obtained in our previous study, and a "responseto-hypertriglyceridemia" hypothesis was proposed that plasma apoA5 elevation could be an adaptive consequence of hypertriglyceridemia and vice versa in these $o b /$ $o b$ mice after treatment with metformin [22]. Similar phenomena were observed in other hypertriglyceridemiarelated diseases, including type 2 diabetes [24], severe hypertriglyceridemia [25], and acute coronary syndrome [26], where plasma apoA5 levels were elevated and positively correlated with TG.

Given that metformin-associated pharmacological actions are mainly dependent on the AMPK signaling pathway [27], we investigated the importance of AMPK in metformin-induced down-regulation of apoA5. As expected, metformin treatment led to AMPK phosphorylation in animals and hepatocytes, which was accompanied by a significant reduction of apoA5 expression and TG level. However, administration of a selective AMPK inhibitor (compound C) almost eliminated the inhibitory effects of metformin on hepatocyte apoA5 expressions and TG level. Thus, our findings indicate that AMPK activation is required for metformin-mediated inhibition of hepatic apoA5 expression and TG production, which is believed to prevent NAFLD development.

Considering the documented role of LXR $\alpha$ in regulation of hepatic APOA5 mRNA [12], we further investigated the involvement of $\operatorname{LXR} \alpha$ in metformininduced apoA5 down-regulation. Jakel et al found that $\mathrm{LXR} \alpha$ activation resulted in down-regulation of hepatic APOA5 mRNA [12]. By contrast, our study demonstrated that LXR $\alpha$ KD effectively eliminated the inhibitory effects of metformin on hepatocyte apoA5 expression. Actually, the AMPK/LXR $\alpha$ signaling pathway was recently confirmed as important in the antihyperglycemic effects of metformin [13]. In this current study, we observed that addition of the AMPK inhibitor pronouncedly repressed hepatocyte LXR $\alpha$ phosphorylation by metformin. Therefore, this study also implicates the AMPK/LXR $\alpha$ signaling pathway in metformin-induced inhibition of hepatic apoA5 synthesis.

In summary, our findings implicate apoA5 in metformin-mediated amelioration of obesity-induced NAFLD. Metformin down-regulates hepatic apoA5 expression and reductes hepatic TG level, which contributes to prevention of NAFLD development, and involves the AMPK/LXR $\alpha$ signaling pathway. Nevertheless, the clinical significance of our findings, and in particular apoA5 as a potential target for NAFLD management, remains to be investigated in future clinical trials.

\section{MATERIALS AND METHODS}

\section{Animal study}

Experiments were carried out in accordance with a protocol approved by the Institutional Animal Care and Use Committee of Animal Experiment Department of Central South University. Adequate measures were taken to minimize pain or discomfort to experimental animals. Mouse NAFLD model was established as previously described [28]. Animals used in this study were 5-weekold male wild-type (WT) and $o b / o b$ mice on a C57BL/6J genetic background (Animal Center of Nanjing University, Nanjing, China). Animals were housed in specific pathogen-free conditions at constant temperature $(21 \pm$ $\left.1^{\circ} \mathrm{C}\right)$ and humidity $(50 \pm 5 \%)$ under 12 -hour light/dark cycle. All mice received a standard laboratory chow ( $10 \%$ of calories derived from fat, $3.85 \mid \mathrm{kcal} / \mathrm{g}$ ) and water available ad libitum. The results of the food intake were obtained from the mice individually housed in cages; the body weight of the freely available chow in a jar was measured every 3 days, and the daily food intake was calculated. Metformin was dissolved in drinking water and administered by daily oral gavage. After 1 week of adaptation, $o b / o b$ mice were randomized into 3 groups, including NAFLD, low- and high-dose metformin groups, with WT mice as controls ( $\mathrm{n}=10$ each group). The mice in low- and high-dose metformin groups were treated with metformin 50 and $100 \mathrm{mg} / \mathrm{kg}$, respectively. After 4 weeks, animals were fasted overnight and sacrificed under anesthesia with sodium pentobarbital. Blood samples 
were collected in $5 \%$ EDTA tubes stored at $-80^{\circ} \mathrm{C}$ for measurements. Animal livers were excised, with part fixed in $10 \%$ neutral formalin solution, and the remainder frozen immediately in liquid nitrogen and stored at $-80^{\circ} \mathrm{C}$ until use. Measurements of plasma apoA5, lipids, glucose, insulin, ALT, AST, HOMA-IR, and hepatic TG contents were conducted as described previously [13].

\section{Histological analysis of mouse hepatic tissue}

Liver tissues were fixed, dehydrated and embedded in paraffin. Paraffinized tissue sections ( $4 \mu \mathrm{m}$ thickness) were stained with hematoxylin and eosin (H\&E) for microscopic evaluation of the degree of NAFLD. Steatosis score is defined by the sum of scores of macrosteatosis, microstetosis, and hypertrophy [29], with each given a score of 0 to 3 assessed from three randomly selected fields of each liver section. The sum of these scores (steatosis score) was calculated for each fields. The mean score for three fields was calculated as the steatosis score for the specimen, and liver histology of 6 mice per group was examined.

\section{Hepatocyte experiment in vitro}

Murine hepatic IAR20 cells were cultured as previously described [13]. All chemicals were dissolved in DMSO and the final concentration of DMSO in media was maintained at $0.1 \%(\mathrm{v} / \mathrm{v})$. When the cells reached $\sim 50 \%$ confluence, they were cultured in serum-free media and divided into 4 groups: (1) control group, treated with the scrambled control siRNA; (2) metformin group, with 500 $\mu \mathrm{M}$ metformin; (3) AMPK inhibitor group, with $500 \mu \mathrm{M}$ metformin and $40 \mu \mathrm{M}$ compound $\mathrm{C}$, a selective inhibitor of AMPK (Abcam); (4) LXR $\alpha$ KD group, transfected with LXR $\alpha$-targeting siRNA and then treated with 500 $\mu \mathrm{M}$ metformin. At collection, all cells were washed and cellular TG was measured by enzymatic reagents [13].

\section{Transient cell transfection with siRNAs}

As described previously [13], IAR20 cells were transiently transfected with siRNA targeting $\mathrm{LXR} \alpha$ (100 nM) or control, scrambled siRNA (Santa Cruz Biotechnology, California, USA) using Lipofectamine 2000 (Invitrogen, California, USA). The sequences used for LXR $\alpha$ KD were sense 5'-CGU AGC AUU AAG GGA GAG U-3' and antisense 5'-ACU CUC CCU UAA UGC UAC G-3'. The sequences for the control siRNA were sense 5'-CCU ACG CCA CCA AUU UCG U-3' and antisense 5'-ACG AAA UUG GUG GCG UAG G-3'. The transfected cells were stabilized for 48 hours before subsequent treatment(s).

\section{Western blot analysis}

As described previously [13], equal protein amounts of whole cell/tissue lysates were loaded into lanes and separated on $10 \%$ SDS-PAGE and transferred onto PVDF membranes. The membranes were then blocked with $5 \%$ nonfat milk in Tris-buffered saline containing $0.05 \%$ Tween 20 (TTBS) at room temperature for 1 hour and incubated at $4^{\circ} \mathrm{C}$ for overnight with a primary antibody diluted $1: 1000$ to $1: 5000$ in 5\% nonfat milk in TTBS. The primary antibodies used were against apoA5 (Abcam, Cambridge, USA), AMPK and p-AMPK (Cell Signaling, Massachusetts, USA), LXR $\alpha$ and p-LXR $\alpha$ (Abcam, Cambridge, USA). After incubation with a HRP-conjugated secondary antibody (1:5000), immunoreactive bands were visualized using an Enhanced Chemiluminescence Plus Kit (GE Healthcare, Pittsburgh, USA), followed by exposure to X-ray film.

\section{Statistical analysis}

All statistical calculations were performed with SPSS 15.0 statistical software package (SPSS). Data are presented as mean \pm SD. When two groups were compared, the significance was evaluated by unpaired Student t-test; when multiple groups were compared, the significance was evaluated by one-way ANOVA followed by the test of Student-Newman-Keuls. Coefficients of correlation (r) were calculated by the Pearson correlation analysis. Results were considered statistically significant at two-sided $p<0.05$.

\section{Abbreviations}

ApoA5, apolipoprotein A5; NAFLD, non-alcoholic fatty liver disease; $\operatorname{LXR} \alpha$, liver $\mathrm{X}$ receptor $\alpha$; AMPK, AMP-activated protein kinase; TG, triglyceride; ALT, alanine aminotransferase; AST, aspartate aminotransferase; HOMA-IR, homeostasis model assessment of insulin resistance; H\&E, hematoxylin and eosin; KD, knockdown; TTBS, Tris-buffered saline containing $0.05 \%$ Tween 20 .

\section{Author contributions}

Conceived and designed the experiments: XSH. Performed the experiments: MJL WD RL YQZ YY LZC SHW. Analyzed the data: MJL WD MJS RL XSH. Wrote the paper: MJS RL XSH.

\section{ACKNOWLEDGMENTS}

The authors thank Min $\mathrm{Hu}$ and Sai Nie for their superior technical and scientific assistance, and thank Shuhui Wang Lorkowski for her language modification of this manuscript.

\section{CONFLICTS OF INTEREST}

The authors have no conflicts of interest to declare in relation to this study. 


\section{FUNDING}

This work was supported by National Natural Science Foundation for Young Programs of China (No. 81000121; No. 81500358) and Natural Science Foundation of Hunan Province (No. 2017JJ2351).

\section{REFERENCES}

1. Williams CD, Stengel J, Asike MI, Torres DM, Shaw J, Contreras M, Landt CL, Harrison SA. Prevalence of nonalcoholic fatty liver disease and nonalcoholic steatohepatitis among a largely middle-aged population utilizing ultrasound and liver biopsy: a prospective study. Gastroenterology. 2011; 140: 124-31.

2. Bellentani S, Saccoccio G, Masutti F, Crocè LS, Brandi G, Sasso F, Cristanini G, Tiribelli C. Prevalence of and risk factors for hepatic steatosis in Northern Italy. Ann Intern Med. 2000; 132: 112-7.

3. Li L, Liu DW, Yan HY, Wang ZY, Zhao SH, Wang B. Obesity is an independent risk factor for non-alcoholic fatty liver disease: evidence from a meta-analysis of 21 cohort studies. Obes Rev. 2016; 17: 510-19.

4. Garinis GA, Fruci B, Mazza A, De Siena M, Abenavoli S, Gulletta E, Ventura V, Greco M, Abenavoli L, Belfiore A. Metformin versus dietary treatment in nonalcoholic hepatic steatosis: a randomized study. Int J Obes (Lond). 2010; 34: 1255-64.

5. Pennacchio LA, Olivier M, Hubacek JA, Cohen JC, Cox DR, Fruchart JC, Krauss RM, Rubin EM. An apolipoprotein influencing triglycerides in humans and mice revealed by comparative sequencing. Science. 2001; 294: 169-73.

6. Priore Oliva C, Pisciotta L, Li Volti G, Sambataro MP, Cantafora A, Bellocchio A, Catapano A, Tarugi P, Bertolini S, Calandra S. Inherited apolipoprotein A-V deficiency in severe hypertriglyceridemia. Arterioscler Thromb Vasc Biol. 2005; 25: 411-7.

7. Shu X, Chan J, Ryan RO, Forte TM. Apolipoprotein A-V association with intracellular lipid droplets. J Lipid Res. 2007; 48: 1445-50.

8. Shu X, Nelbach L, Ryan RO, Forte TM. Apolipoprotein A-V associates with intrahepatic lipid droplets and influences triglyceride accumulation. Biochim Biophys Acta. 2010; 1801: 605-8.

9. Gao X, Forte TM, Ryan RO. Influence of apolipoprotein $\mathrm{A}-\mathrm{V}$ on hepatocyte lipid droplet formation. Biochem Biophys Res Commun. 2012; 427: 361-5.

10. Ress C, Moschen AR, Sausgruber N, Tschoner A, Graziadei I, Weiss H, Schgoer W, Ebenbichler CF, Konrad RJ, Patsch JR, Tilg H, Kaser S. The role of apolipoprotein A5 in non-alcoholic fatty liver disease. Gut. 2011; 60: 985-91.

11. Feng Q, Baker SS, Liu W, Arbizu RA, Aljomah G, Khatib M, Nugent CA, Baker RD, Forte TM, Hu Y, Zhu L.
Increased apolipoprotein A5 expression in human and rat non-alcoholic fatty livers. Pathology. 2015; 47: 341-8.

12. Jakel H, Nowak M, Moitrot E, Dehondt H, Hum DW, Pennacchio LA, Fruchart-Najib J, Fruchart JC. The liver $\mathrm{X}$ receptor ligand T0901317 down-regulates APOA5 gene expression through activation of SREBP-1c. J Biol Chem. 2004; 279: 45462-9.

13. Cho K, Chung JY, Cho SK, Shin HW, Jang IJ, Park JW, Yu KS, Cho JY. Antihyperglycemic mechanism of metformin occurs via the AMPK/LXR $\alpha /$ POMC pathway. Sci Rep. 2015; 5: 8145.

14. Lin HZ, Yang SQ, Chuckaree C, Kuhajda F, Ronnet G, Diehl AM. Metformin reverses fatty liver disease in obese, leptin-deficient mice. Nat Med. 2000; 6: 998-1003.

15. Al-Barazanji K, McNulty J, Binz J, Generaux C, Benson W, Young A, Chen L. Synergistic effects of a GPR119 agonist with metformin on weight loss in diet-induced obese mice. J Pharmacol Exp Ther. 2015; 353: 496-504.

16. Cifarelli V, Lashinger LM, Devlin KL, Dunlap SM, Huang J, Kaaks R, Pollak MN, Hursting SD. Metformin and rapamycin reduce pancreatic cancer growth in obese prediabetic mice by distinct MicroRNA-regulated mechanisms. Diabetes. 2015; 64: 1632-42.

17. Seifarth C, Schehler B, Schneider HJ. Effectiveness of metformin on weight loss in non-diabetic individuals with obesity. Exp Clin Endocrinol Diabetes. 2013; 121: 27-31.

18. Jarskog LF, Hamer RM, Catellier DJ, Stewart DD, Lavange L, Ray N, Golden LH, Lieberman JA, Stroup TS; METS Investigators. Metformin for weight loss and metabolic control in overweight outpatients with schizophrenia and schizoaffective disorder. Am J Psychiatry. 2013; 170: 1032-40.

19. Diabetes Prevention Program Research Group. Longterm safety, tolerability, and weight loss associated with metformin in the Diabetes Prevention Program Outcomes Study. Diabetes Care. 2012; 35: 731-7.

20. Mazza A, Fruci B, Garinis GA, Giuliano S, Malaguarnera $\mathrm{R}$, Belfiore A. The role of metformin in the management of NAFLD. Exp Diabetes Res. 2012; 2012: 716404.

21. UK Prospective Diabetes study Group. Effect of intensive blood glucose control with metformin on complications in overweight patients with type 2 diabetes (UKPDS 34). Lancet. 1998; 352: 854-65.

22. Li R, Chen LZ, Zhao W, Zhao SP, Huang XS. Metformin ameliorates obesity-associated hypertriglyceridemia in mice partly through the apolipoprotein A5 pathway. Biochem Biophys Res Commun. 2016; 478: 1173-8.

23. Perry RJ, Samuel VT, Petersen KF, Shulman GI. The role of hepatic lipids in hepatic insulin resistance and type 2 diabetes. Nature. 2014; 510: 84-91.

24. Talmud PJ, Cooper JA, Hattori H, Miller IP, Miller GJ, Humphries SE. The apolipoprotein A-V genotype and plasma apolipoprotein $\mathrm{A}-\mathrm{V}$ and triglyceride levels: prospective risk of type 2 diabetes. Results from the Northwick Park Heart Study II. Diabetologia. 2006; 49: 2337-40. 
25. Schaap FG, Nierman MC, Berbée JF, Hattori H, Talmud PJ, Vaessen SF, Rensen PC, Chamuleau RA, Kuivenhoven JA, Groen AK. Evidence for a complex relationship between apoA-V and apoC-III in patients with severe hypertriglyceridemia. J Lipid Res. 2006; 47: 2333-9.

26. Huang XS, Zhao SP, Zhang Q, Bai L, Hu M. Association of plasma apolipoprotein AV with lipid profiles in patients with acute coronary syndrome. Atherosclerosis. 2009; 204: e99-102.

27. Zhou G, Myers R, Li Y, Chen Y, Shen X, Fenyk-Melody J, Wu M, Ventre J, Doebber T, Fujii N, Musi N, Hirshman MF, Goodyear LJ, et al. Role of AMP-activated protein kinase in mechanism of metformin action. J Clin Invest. 2001; 108: 1167-74.

28. Liang W, Menke AL, Driessen A, Koek GH, Lindeman JH, Stoop R, Havekes LM, Kleemann R, van den Hoek AM. Establishment of a general NAFLD scoring system for rodent models and comparison to human liver pathology. PLoS One. 2014; 9: e115922.

29. Inafuku $\mathrm{M}$, Matsuzaki $\mathrm{G}, \mathrm{Oku} H$. Intravenous Mycobacterium bovis Bacillus Calmette-Guérin ameliorates nonalcoholic fatty liver disease in obese, diabetic ob/ob Mice. PLoS One. 2015; 10: e0128676. 